\title{
Proton-Catalyzed Hydroamination and Hydroarylation Reactions of Anilines and Alkenes. A Dramatic Effect of Counteranions on Reaction Efficiency.
}

\author{
Laura L. Anderson, John Arnold*, Robert G. Bergman* \\ Department of Chemistry, University of California Berkeley, Berkeley, California, 94720 \\ Supporting Information
}

\section{Experimental Details}

General Information. Syntheses of anilinium salts and $\mathbf{3}$ were carried out in an inert atmosphere $\left(\mathrm{N}_{2}\right)$ glovebox at $10{ }^{\circ} \mathrm{C}$ or using standard Schlenk and high vacuum techniques. All glassware used in these catalyst syntheses was dried in an oven at $150{ }^{\circ} \mathrm{C}$ for at least $12 \mathrm{~h}$ prior to use or was flame dried under reduced pressure. All of the catalysts shown in Table 1 were stored in an inert atmosphere $\left(\mathrm{N}_{2}\right)$ glovebox at $10{ }^{\circ} \mathrm{C}$ to prevent prolonged exposure to moisture. Catalytic reactions were set up both inside an inert atmosphere $\left(\mathrm{N}_{2}\right)$ glovebox at $10{ }^{\circ} \mathrm{C}$ and on the benchtop at $25{ }^{\circ} \mathrm{C}$. Larger scale reactions $(\sim 1$ mmol of substrate) were run in teflon-sealed Kontes reaction vessels and smaller scale reactions $(\sim 0.3$ mmol of substrate) were run in flame-sealed NMR tubes. Oil baths were calibrated using analytical mercury thermometers purchased from VWR that are calibrated from $0-500^{\circ} \mathrm{C}$. Although some catalytic reactions were set-up under an inert atmosphere and some were set up on the benchtop, no difference in reactivity was observed. Sealed NMR tubes were prepared by attaching the NMR tube directly to a Kontes high-vacuum stopcock via a cajon ultra-torr reducing union, then flame-sealing on a vacuum line. NMR tube reactions were monitored with sealed capillary tube external standards that were filled with a mixture of $\mathrm{C}_{6} \mathrm{D}_{6}$ and $\mathrm{CH}_{2} \mathrm{Cl}_{2}$. Dodecane was used as an internal standard for reactions that were monitored by GC-MS. Flash chromatography was carried out on Merck 60 silica gel (32-63 $\mu \mathrm{m})$ and fractions were analyzed on Whatman aluminum-backed, $250 \mu \mathrm{m}$, silica gel TLC plates with ceric 
ammonium molybdate stain. $\quad{ }^{1} \mathrm{H}$ and ${ }^{13} \mathrm{C}\left\{{ }^{1} \mathrm{H}\right\}$ NMR spectra were recorded on Bruker AVQ-400 (400 $\mathrm{MHz}$ ), AV-400 (400 MHz), and DRX-500 (500 MHz) spectrometers as indicated. ${ }^{19} \mathrm{~F}$ spectra were recorded on a Bruker AVQ-400 (400 MHz) spectrometer and ${ }^{11} \mathrm{~B}$ spectra were recorded on a Bruker DRX-500 (500 MHz) spectrometer. ${ }^{1} \mathrm{H}$ NMR chemical shifts $(\delta)$ are reported in parts per million $(\mathrm{ppm})$ relative to residual protiated solvent $\left(\mathrm{C}_{6} \mathrm{D}_{6}, 7.15 ; \mathrm{C}_{6} \mathrm{D}_{5} \mathrm{Cl}, 6.99(\mathrm{o}-\mathrm{CH})\right)$ or an external standard of $\mathrm{CH}_{2} \mathrm{Cl}_{2}$ in $\mathrm{C}_{6} \mathrm{D}_{6}$ (4.27). Data are reported as follows: $(\mathrm{s}=$ singlet, $\mathrm{d}=$ doublet, $\mathrm{t}=$ triplet, $\mathrm{q}=$ quartet, $\mathrm{m}=$ multiplet; coupling constant(s) in Hz; integration; assignment). Chemical shifts were assigned with the aid of DEPT 45, 90 and 135 experiments where necessary. Gas Chromatograph-Mass Spectroscopy (GCMS) data were obtained using an Agilent Technologies Instrument 6890N GC (column \#HP-5MS, 30.0m x $250 \mu \mathrm{m}$ x $0.25 \mu \mathrm{m}$ calibrated) and 5973N MS. Gas chromatography data were obtained using an Agilent Technologies Instrument 6850 with an HP-1 column. Elemental analyses were performed at the UCBerkeley Microanalytical Facility with a Perkin Elmer 2400 Series II CHNO/S Analyzer. High resolution mass spectra were obtained at the UC-Berkeley Mass Spectrometry Facility with a VG ProSpec Mass Spectrometer using electron impact ionization at $70 \mathrm{eV}$.

Materials. The diethylether and methylene chloride (Fisher) used for the syntheses of the anilinium salts and $\mathbf{3}$ were dried and purified by passage through a column of activated alumina under $\mathrm{N}_{2}$ pressure, sparging with $\mathrm{N}_{2}$, and storage over $4 \AA$ sieves. ${ }^{1}$ Benzene was used as received from the vendor (Fisher) or purified by passage through a column of activated alumina under $\mathrm{N}_{2}$ pressure, sparging with $\mathrm{N}_{2}$, and storage over $4 \AA$ sieves. ${ }^{1}$ Chlorobenzene was used as received from the vendor (Aldrich) or dried over $\mathrm{CaH}_{2}$, vacuum transferred, and degassed with 4 freeze-evacuation-thaw cycles. Benzene- $d_{6}$ (Cambridge Isotope Laboratories) was used as received or vacuum transferred from a purple solution of sodium/benzophenone. Chlorobenzene- $d_{5}$ (Cambridge Isotope Laboratories) was used as received or vacuum transferred from $\mathrm{CaH}_{2}$ and degassed with 4 freeze-evacuation-thaw cycles. No difference in 
reactivity was observed for catalytic reactions run in dried and degassed solvent or solvent used as received from the vendor. The $1 \mathrm{M}$ solution of $\mathrm{HCl}$ in diethylether, $\mathrm{HOTf}, \mathrm{HNTf}_{2}, \mathrm{NaBPh}_{4}, 1,3-$ cyclohexadiene, $p$-methoxystyrene and $p$-chlorostyrene were purchased from Aldrich and used as received. Norbornene (Aldrich) was vacuum transferred. Aniline, 3,5-bis(trifluoromethyl)aniline, 3chloroaniline, $\mathrm{N}$-methylaniline, and 2,6-dimethylaniline (Aldrich) were distilled from $\mathrm{CaH}_{2}$, degassed with 4 freeze-evacuation-thaw cycles, and stored in the glovebox. Anisidine (Aldrich) was sublimed and stored in the glovebox. $p$-Chloroaniline (Aldrich) was crystallized from hot hexanes and stored in the glovebox. Cis-cyclooctene and cyclopentene (Aldrich) were distilled from $\mathrm{CaH}_{2}$, degassed with 4 freezeevacuation-thaw cycles and stored in the glovebox. Dodecane was also distilled from $\mathrm{CaH}_{2}$. The hexane and ethylacetate that were used for chromatography were used as received from the vendor (VWR). $\mathrm{Ph}_{3} \mathrm{CB}\left(\mathrm{C}_{6} \mathrm{~F}_{5}\right)_{4}$ and $\mathrm{LiB}\left(\mathrm{C}_{6} \mathrm{~F}_{5}\right)_{4} \cdot\left(\mathrm{Et}_{2} \mathrm{O}\right)_{2.5}$ were synthesized according to literature procedures. ${ }^{2}$

Preparation of $\left[\mathbf{C}_{6} \mathbf{H}_{5} \mathbf{N H}_{3}\right][\mathbf{O T f}]$. In the glovebox, a Schlenk tube was charged with $3.42 \mathrm{~g}(36.7$ mmol) of aniline and $60 \mathrm{~mL}$ of $\mathrm{CH}_{2} \mathrm{Cl}_{2}$. The Schlenk tube was then sealed, taken out of the glovebox, flushed onto a Schlenk line, and cooled to $0{ }^{\circ} \mathrm{C}$ with an ice/water bath. While stirring, $3.30 \mathrm{~mL}(36.7$ mmol) of HOTf were added via syringe. A white precipitate appeared immediately. The reaction mixture was allowed to stir overnight at room temperature. The volatile materials were then removed under reduced pressure to give a white solid. The Schlenk tube was taken back into the glovebox under vacuum. The white solid was isolated $7.09 \mathrm{~g}\left(80 \%\right.$ yield). ${ }^{1} \mathrm{H}$ NMR (THF- $\left.d_{8}, 400 \mathrm{MHz}\right) \delta 9.529(\mathrm{~s}, 3 \mathrm{H}$, $\left.\mathrm{N} H_{3}\right) ;$ 7.67-7.30 (m, 5H, ArH). ${ }^{13} \mathrm{C}\left\{{ }^{1} \mathrm{H}\right\} \mathrm{NMR}\left(\mathrm{THF}-d_{8}, 500 \mathrm{MHz}\right) \delta 131.9,130.7,129.7,124.2,119.1$ $\left(\mathrm{q},{ }^{1} J_{\mathrm{C}-\mathrm{F}}=317.5 \mathrm{~Hz}\right) .{ }^{19} \mathrm{~F}$ NMR $\left(\mathrm{THF}-d_{8}, 376 \mathrm{MHz}\right) \delta$-79.4. Anal. Calcd. C, 34.57; H, 3.32; N, 5.76. Found C, 34.58; H, 3.22; N, 5.76.

Preparation of $\left[\mathbf{H}\left(\mathbf{E t}_{\mathbf{2}} \mathbf{O}\right)_{2.5}\right]\left[\mathbf{B}\left(\mathbf{C}_{\mathbf{6}} \mathbf{F}_{4}\right)_{4}\right]$. In the glovebox, a Schlenk tube was charged with $1.09 \mathrm{~g}$ $(1.25 \mathrm{mmol})$ of $\mathrm{LiB}\left(\mathrm{C}_{6} \mathrm{~F}_{5}\right)_{4} \cdot 2.5 \mathrm{Et}_{2} \mathrm{O}$ and approximately $60 \mathrm{~mL}$ of $\mathrm{Et}_{2} \mathrm{O}$. The schlenk tube was then sealed, taken out of the box and flushed onto a line. The $\mathrm{LiB}\left(\mathrm{C}_{6} \mathrm{~F}_{5}\right)_{4}$ solution was then cooled to $0{ }^{\circ} \mathrm{C}$ with 
an ice/water bath and $1.25 \mathrm{~mL}(1.25 \mathrm{mmol})$ of a $1.0 \mathrm{M}$ solution of $\mathrm{HCl}$ in $\mathrm{Et}_{2} \mathrm{O}$ was added via syringe.

The reaction mixture was then allowed to stir and warm to room temperature for $4 \mathrm{~h}$. Volatile materials from the reaction mixture were then removed under reduced pressure to give a white solid. The reaction flask was then taken into the glovebox. The white solid was washed with ether and quickly filtered to separate the $\mathrm{LiCl}$ byproduct. The filtrate was then placed in the $-40{ }^{\circ} \mathrm{C}$ freezer overnight. Clear, needlelike crystals formed. The bulk of the solvent was carefully removed via pipet and the remaining volatile materials were removed under reduced pressure to give $510 \mathrm{mg}$ (47\% yield) of the white solid, $\left[\mathrm{H}\left(\mathrm{Et}_{2} \mathrm{O}\right)_{2}\right]\left[\mathrm{B}\left(\mathrm{C}_{6} \mathrm{~F}_{5}\right)_{4}\right] .{ }^{1} \mathrm{H} \mathrm{NMR}\left(\mathrm{C}_{6} \mathrm{D}_{5} \mathrm{Cl}, 500 \mathrm{MHz}\right) \delta 3.21\left(\mathrm{q}, J=7.0 \mathrm{~Hz}, 10 \mathrm{H}, \mathrm{CH}_{2}\right), 0.88(\mathrm{t}, J=7.0 \mathrm{~Hz}$, $15 \mathrm{H}, \mathrm{CH}) .{ }^{13} \mathrm{C}\left\{{ }^{1} \mathrm{H}\right\} \operatorname{NMR}\left(\mathrm{C}_{6} \mathrm{D}_{5} \mathrm{Cl}, 500 \mathrm{MHz}\right) \delta 150.6\left(\mathrm{~m}, \mathrm{Ar}_{\mathrm{F}} C\right), 147.0\left(\mathrm{~m}, \mathrm{Ar}_{\mathrm{F}} C\right), 139.8(\mathrm{~m}$, ipso$\left.\operatorname{Ar}_{\mathrm{F}} C\right), 138.2\left(\mathrm{~m}, \operatorname{Ar}_{\mathrm{F}} C\right), 135.3\left(\mathrm{~m}, \mathrm{Ar}_{\mathrm{F}} C\right), 55.1,14.1 .{ }^{19} \mathrm{~F} \mathrm{NMR}\left(\mathrm{C}_{6} \mathrm{D}_{5} \mathrm{Cl}, 376 \mathrm{MHz}\right) \delta-131.7,-162.3,-$ 166.2. ${ }^{11} \mathrm{~B}$ NMR $\left(\mathrm{C}_{6} \mathrm{D}_{5} \mathrm{Cl}, 160 \mathrm{MHz}\right) \delta$-16.6. Anal. Calcd. C, 47.19; H, 3.03. Found C, 47.29; H, 3.01.

Preparation of $\left[\mathbf{C}_{6} \mathbf{H}_{5} \mathbf{N H}_{3}\right]\left[\mathbf{B}\left(\mathbf{C}_{6} \mathbf{F}_{5}\right)_{4}\right] \cdot \mathbf{E} \mathbf{t}_{2} \mathbf{O}$. In the glovebox, a vial was charged with $318 \mathrm{mg}(1.31$ mmol) of anilinium triflate, $1.14 \mathrm{~g}(1.31 \mathrm{mmol})$ of $\mathrm{LiB}\left(\mathrm{C}_{6} \mathrm{~F}_{5}\right)_{4} \cdot 2.5 \mathrm{Et}_{2} \mathrm{O}$, and $10 \mathrm{~mL}$ of $\mathrm{CH}_{2} \mathrm{Cl}_{2}$. The mixture was allowed to stir for $8 \mathrm{~h}$ at $10{ }^{\circ} \mathrm{C}$. The reaction mixture was then allowed to settle. The supernatant was decanted via pipet and filtered through a glass fiber filter pipet plug in order to separate the insoluble LiOTf. Volatile materials were then removed under reduced pressure to give a clear oil that was washed with $2 \times 5 \mathrm{~mL}$ of pentane to give the desired product as a white powder $(982 \mathrm{mg}, 75 \%)$. ${ }^{1} \mathrm{H}$ $\operatorname{NMR}\left(\mathrm{C}_{6} \mathrm{D}_{5} \mathrm{Cl}, 400 \mathrm{MHz}\right) \delta 7.41(\mathrm{~s}, 3 \mathrm{H}, \mathrm{NH}), 7.11(\mathrm{~m}, 3 \mathrm{H}, \mathrm{Ar} H), 6.73(\mathrm{~m}, 2 \mathrm{H}, \mathrm{Ar} H), 3.18(\mathrm{q}, J=7.2$ $\left.\mathrm{Hz}, 4 \mathrm{H}, \mathrm{CH}_{2}\right), 0.82(\mathrm{t}, J=7.2 \mathrm{~Hz}, 6 \mathrm{H}, \mathrm{CH}) .{ }^{13} \mathrm{C}\left\{{ }^{1} \mathrm{H}\right\}\left(\mathrm{C}_{6} \mathrm{D}_{5} \mathrm{Cl}, 100 \mathrm{MHz}\right) \delta 149.8\left(\mathrm{~m}, \operatorname{Ar}_{\mathrm{F}} C, 147.4(\mathrm{~m}\right.$, $\left.\operatorname{Ar}_{\mathrm{F}} C\right), 139.8\left(\mathrm{~m}\right.$, ipso- $\left.\mathrm{Ar}_{\mathrm{F}} C\right), 138.0\left(\mathrm{~m}, \mathrm{Ar}_{\mathrm{F}} C\right), 135.5\left(\mathrm{~m}, \mathrm{Ar}_{\mathrm{F}} C\right), 130.7,130.1,121.1 .{ }^{19} \mathrm{~F} \mathrm{NMR}\left(\mathrm{C}_{6} \mathrm{D}_{5} \mathrm{Cl}\right.$, $376 \mathrm{MHz}) \delta-131.9,-161.2,-165.4 .{ }^{11} \mathrm{~B}\left(\mathrm{C}_{6} \mathrm{D}_{5} \mathrm{Cl}, 164.5 \mathrm{MHz}\right) \delta$-16.6. Anal. Calcd. C, 48.20; H, 2.14; $\mathrm{N}$, 1.65. Found C, 48.34; H, 2.09; N, 1.66. 
Preparation of $\left[\mathbf{C}_{6} \mathbf{H}_{5} \mathbf{N H}_{3}\right]\left[\mathrm{BPh}_{4}\right]$. This reagent was prepared by an analogous salt metathesis method to a previously reported preparation. ${ }^{3}$ In the glovebox, a vial was charged with $311 \mathrm{mg}$ of anilinium triflate, $437 \mathrm{mg}$ of $\mathrm{NaBPh}_{4}$, and $10 \mathrm{~mL}$ of $\mathrm{CH}_{2} \mathrm{Cl}_{2}$. The mixture was allowed to stir for $8 \mathrm{~h}$ at 10 ${ }^{\circ} \mathrm{C}$ in a vial covered with foil to keep out light. The reaction mixture was then allowed to settle. The reaction mixture was filtered through a frit in a dark glovebox. The filtrate was pumped down to give a white solid. (341 mg, 64\%). ${ }^{1} \mathrm{H}$ NMR $\left(\mathrm{THF}_{-} \mathrm{d}_{8}, 400 \mathrm{MHz}\right) \delta 7.31(\mathrm{~m}, 3 \mathrm{H}, \mathrm{NH}$ ) $, 7.26(\mathrm{~m}, 6 \mathrm{H}, \mathrm{ArH}), 7.24-$ $6.99(\mathrm{~m}, 12 \mathrm{H}, \mathrm{Ar} H), 6.73(\mathrm{~m}, 2 \mathrm{H}, \mathrm{Ar} H) .{ }^{1} \mathrm{H}$ NMR resonances match literature values. ${ }^{3}$

General experimental procedure for the experiments in Table 1. A vial was charged with an accurately weighed amount of norbornene that was $\sim 25 \mathrm{mg}(\sim 0.27 \mathrm{mmol})$ for each reaction. The norbornene was dissolved in $\sim 0.5 \mathrm{~mL}$ of benzene. The resulting solution was mixed with 5 equivalents of aniline, ${ }^{4} 1$ equivalent of dodecane and $5 \mathrm{~mol} \%$ of catalyst in the order given. The reaction mixture was then transferred to an NMR tube and flame-sealed. The NMR tube was placed in an oil bath calibrated to $135{ }^{\circ} \mathrm{C}$ for $27 \mathrm{~h}$. The NMR tube was then opened and the contents mixed with $\sim 3 \mathrm{~mL}$ of ether before analysis by $\mathrm{GC} / \mathrm{MS}$.

General experimental procedure for the experiments in Table 2. A vial was charged with an accurately weighed amount of norbornene that was $\sim 80 \mathrm{mg}(\sim 0.86 \mathrm{mmol})$ for each reaction. The norbornene was dissolved in 4-5 $\mathrm{mL}$ of benzene. The resulting solution was mixed with 5 equivalents ${ }^{4}$ of the aniline and $5 \mathrm{~mol} \%$ of 4 . The reaction mixture was transferred to a reaction vessel, sealed with a Kontes teflon stopper and placed in an oil bath calibrated to $135{ }^{\circ} \mathrm{C}$ for $48 \mathrm{~h}$. The reaction mixture was then transferred to a vial and volatile materials were removed by rotary evaporation to give an oil. The oil was purified by flash chromatography. 
NMR reference structures for compounds in Table 2.

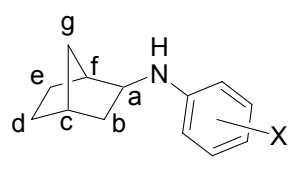

A

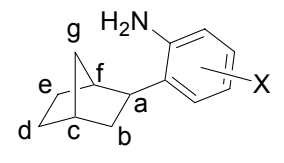

B

Table 2, entry 1: Flash chromatography conditions: Gradient from 5\% ethylacetate/hexane to $10 \%$ ethylacetate/hexane. Combined yield of 6 and 7: $144 \mathrm{mg}$ (83.7\%). Ratio of 6:7, 1:1.

Table 2, entry 1, 6: ${ }^{1} \mathrm{H}$ NMR $\left(\mathrm{C}_{6} \mathrm{D}_{6}, 400 \mathrm{MHz}\right) \delta 7.20(\mathrm{t}, J=7.2 \mathrm{~Hz}, 2 \mathrm{H}, m-\mathrm{Ar} H), 6.77(\mathrm{t}, J=7.2 \mathrm{~Hz}$, 1H, $p-\operatorname{Ar} H), 6.49(\mathrm{~d}, J=7.6 \mathrm{~Hz}, 2 \mathrm{H}, o-\operatorname{Ar} H), 3.09$ (bs, 1H, NH), $2.99\left(\mathrm{~m}, 1 \mathrm{H}, H_{a}\right), 2.07\left(\mathrm{bs}, 1 \mathrm{H}, H_{f}\right), 2.00$ $\left(\mathrm{bs}, 1 \mathrm{H}, H_{c}\right), 1.48\left(\mathrm{~m}, 1 \mathrm{H}\right.$, exo- $\left.H_{b}\right), 1.28\left(\mathrm{~m}, 2 \mathrm{H}\right.$, endo- $H_{b}$, exo- $\left.H_{e}\right), 1.21\left(\mathrm{~m}, 1 \mathrm{H}\right.$, syn- $\left.H_{g}\right), 0.92(\mathrm{~m}, 3 \mathrm{H}$, endo- $\left.H_{e}, H_{d}\right), 0.86\left(\mathrm{~m}, 1 \mathrm{H}\right.$, anti- $\left.H_{g}\right) .{ }^{13} \mathrm{C}\left\{{ }^{1} \mathrm{H}\right\} \operatorname{NMR}\left(\mathrm{C}_{6} \mathrm{D}_{6}, 100 \mathrm{MHz}\right) \delta 147.8,129.2,117.0,113.3,56.4$, 41.1, 40.7, 35.6, 35.2, 28.5, 26.2. Anal. Calcd. C, 83.37; H, 9.15; N, 7.48. Found. C, 83.76; H, 9.50; N, 7.43. Yield: $68.6 \mathrm{mg}(40.0 \%)$.

Table 2, entry 1, 7: ${ }^{1} \mathrm{H}$ NMR $\left(\mathrm{C}_{6} \mathrm{D}_{6}, 400 \mathrm{MHz}\right) \delta 7.09(\mathrm{~d}, J=7.6 \mathrm{~Hz}, 1 \mathrm{H}, \mathrm{Ar} H), 7.04(\mathrm{t}, J=7.6 \mathrm{~Hz}$, 1H, ArH), $6.80(\mathrm{t}, J=7.6 \mathrm{~Hz}, 1 \mathrm{H}, \operatorname{Ar} H), 6.45(\mathrm{t}, J=7.6 \mathrm{~Hz}, 1 \mathrm{H}, \operatorname{Ar} H), 2.98(\mathrm{bs}, 1 \mathrm{H}, \mathrm{NH}), 2.33(\mathrm{~m}, 2 \mathrm{H}$, $\left.H_{a}, H_{f}\right), 2.15\left(\mathrm{bs}, 1 \mathrm{H}, H_{c}\right), 1.52-1.43\left(\mathrm{~m}, 5 \mathrm{H}\right.$, exo- $H_{b}$, endo- $H_{b}$, exo- $H_{d}$, exo- $\left.H_{e}, s y n-H_{g}\right), 1.11(\mathrm{~m}, 2 \mathrm{H}$, endo- $H_{d}$, endo- $\left.H_{e}\right), 1.04\left(\mathrm{~d}, 1 \mathrm{H}\right.$, anti- $\left.H_{g}\right) .{ }^{13} \mathrm{C}\left\{{ }^{1} \mathrm{H}\right\} \mathrm{NMR}\left(\mathrm{C}_{6} \mathrm{D}_{6}, 100 \mathrm{MHz}\right) \delta 144.7,131.2,126.6,125.5$, 118.4, 115.6, 42.2, 40.6, 37.9, 37.2, 36.4, 30.4, 29.6. Anal. Calcd. C, 83.37; H, 9.15; N, 7.48. Found C, 83.02; H, 9.20; N, 7.48. Yield: $75.0 \mathrm{mg}(43.7 \%)$.

Neat reaction for Table 2, entry 1: If aniline is used as a solvent, identical products are obtained in lower yield. Yield of 6 from neat reation: $41.1 \mathrm{mg}$ (22.5\%). Yield of 7 from neat reaction: $43.7 \mathrm{mg}$ (23.9\%). Combined yield of 6 and 7 from neat reaction: $84.8 \mathrm{mg}$ (46.4\%). Ratio of 6:7 from neat reaction; $1: 1$.

Table 2, entry 2, 6: Flash chromatography conditions: 5\% ethylacetate/hexane. ${ }^{1} \mathrm{H}$ NMR $\left(\mathrm{C}_{6} \mathrm{D}_{6}, 400\right.$ MHz) $\delta 7.12(\mathrm{~d}, J=8.8 \mathrm{~Hz}, 2 \mathrm{H}, \mathrm{Ar} H), 6.14(\mathrm{~d}, J=8.8 \mathrm{~Hz}, 2 \mathrm{H}, \mathrm{Ar} H), 2.96(\mathrm{bs}, 1 \mathrm{H}, \mathrm{NH}), 2.78(\mathrm{~m}, 1 \mathrm{H}$, 
$\left.H_{a}\right), 1.98\left(\mathrm{bs}, 1 \mathrm{H}, H_{f}\right), 1.93\left(\mathrm{bs}, 1 \mathrm{H}, H_{c}\right), 1.39\left(\mathrm{~m}, 1 \mathrm{H}\right.$, exo- $\left.H_{b}\right), 1.26\left(\mathrm{~m}, 2 \mathrm{H}\right.$, endo- $H_{b}$, exo- $\left.H_{e}\right), 1.13(\mathrm{~m}$, $1 \mathrm{H}$, syn- $\left.H_{g}\right), 0.89\left(\mathrm{~m}, 3 \mathrm{H}\right.$, endo- $\left.H_{e}, H_{d}\right), 0.76\left(\mathrm{~m}, 1 \mathrm{H}\right.$, anti- $\left.H_{g}\right) .{ }^{13} \mathrm{C}\left\{{ }^{1} \mathrm{H}\right\} \mathrm{NMR}\left(\mathrm{C}_{6} \mathrm{D}_{6}, 100 \mathrm{MHz}\right) \delta 146.4$, 129.3, 121.7, 114.4, 56.6, 41.2, 40.8, 35.8, 35.4, 28.6, 26.4. Anal. Calcd. C, 70.42; H, 7.27; N, 6.32. Found C, 70.49; H, 7.22; N, 6.37. Yield: $108 \mathrm{mg}(55.7 \%)$.

Reaction catalyzed by 5 for Table 2, entry 2, 6: The same product was obtained when 5 was used as a catalyst. The reaction catalyzed by 5 was left in the $135{ }^{\circ} \mathrm{C}$ bath for $72 \mathrm{~h}$. Yield: $111 \mathrm{mg}(55.2 \%){ }^{5}$

Table 2, entry 3: Flash chromatography conditions: 10\% ethylacetate/hexane. Combined yield of 6 and 7: $66.6 \mathrm{mg}(33.7 \%)$. Ratio of 6:7, 1:2.

Table 2, entry 3, 6: ${ }^{1} \mathrm{H}$ NMR $\left(\mathrm{C}_{6} \mathrm{D}_{6}, 500 \mathrm{MHz}\right) \delta 6.85(\mathrm{~d}, J=8.0 \mathrm{~Hz}, 2 \mathrm{H}, \mathrm{Ar} H), 6.44(\mathrm{~d}, J=8.0 \mathrm{~Hz}$, 2H, $\operatorname{Ar} H), 3.52(\mathrm{~s}, 3 \mathrm{H}, \mathrm{OMe}), 3.00\left(\mathrm{~m}, 1 \mathrm{H}, H_{a}\right), 2.90(\mathrm{bs}, 1 \mathrm{H}, \mathrm{NH}), 2.11\left(\mathrm{bs}, 1 \mathrm{H}, H_{f}\right), 2.04\left(\mathrm{bs}, 1 \mathrm{H}, H_{c}\right)$, $1.51\left(\mathrm{~m}, 1 \mathrm{H}\right.$, exo- $\left.H_{b}\right), 1.36-1.27\left(\mathrm{~m}, 3 \mathrm{H}\right.$, endo- $H_{b}$, exo- $H_{e}$, syn- $\left.H_{g}\right), 1.09-0.88\left(\mathrm{~m}, 4 \mathrm{H}\right.$, endo- $H_{e}, H_{d}$, anti$\left.H_{g}\right) . \quad{ }^{13} \mathrm{C}\left\{{ }^{1} \mathrm{H}\right\} \operatorname{NMR}\left(\mathrm{C}_{6} \mathrm{D}_{6}, 125 \mathrm{MHz}\right) \delta 152.7,142.5,115.4,114.8,57.6,55.5,41.6,41.2,38.1,35.6$, 29.0, 26.7. Anal. Calcd. C, 77.38; H, 8.81; N, 6.45. Found C, 77.55; H, 9.19; N, 6.38. Yield: 17.0 mg $(8.6 \%)$

Table 2, entry 3, 7: ${ }^{1} \mathrm{H}$ NMR $\left(\mathrm{C}_{6} \mathrm{D}_{6}, 500 \mathrm{MHz}\right) \delta 6.94(\mathrm{~s}, 1 \mathrm{H}, \mathrm{Ar} H), 6.63(\mathrm{~d}, J=7.5 \mathrm{~Hz}, 1 \mathrm{H}, \mathrm{Ar} H)$, $6.36(\mathrm{~d}, J=7.5 \mathrm{~Hz}, 1 \mathrm{H}, \mathrm{Ar} H), 3.45(\mathrm{~s}, 3 \mathrm{H}, \mathrm{OMe}), 2.80(\mathrm{bs}, 1 \mathrm{H}, \mathrm{NH}), 2.40\left(\mathrm{~m}, 1 \mathrm{H}, H_{a}\right), 2.31\left(\mathrm{bs}, 1 \mathrm{H}, H_{f}\right)$, $2.13\left(\mathrm{bs}, 1 \mathrm{H}, H_{c}\right), 1.55-1.38\left(\mathrm{~m}, 5 \mathrm{H}\right.$, exo- $H_{b}$, endo- $H_{b}$, exo- $H_{d}$, exo- $H_{e}$, syn- $\left.H_{g}\right), 1.13\left(\mathrm{~m}, 2 \mathrm{H}\right.$, endo- $H_{d}$, endo- $\left.H_{e}\right), 1.04\left(\mathrm{~d}, 1 \mathrm{H}\right.$, anti- $\left.H_{g}\right) . \quad{ }^{13} \mathrm{C}\left\{{ }^{1} \mathrm{H}\right\} \operatorname{NMR}\left(\mathrm{C}_{6} \mathrm{D}_{6}, 125 \mathrm{MHz}\right) \delta 153.4,138.6,133.4,116.6,113.1$, 111.2, 55.5, 42.6, 41.0, 38.2, 37.3, 36.6, 30.5, 29.7. Anal. Calcd. C, 77.38; H, 8.81; N, 6.45. Found C, 77.22; H, 8.74; N, 6.73. Yield: $49.6 \mathrm{mg}(25.1 \%)$.

Table 2, entry 4: Under a variety of chromatography conditions, both products of this reaction coeluted with excess aniline from the reaction mixture. Therefore, 6 and 7 were not separated and isolated 
from the reaction mixture. A combined yield of 50\% (14\% 6 and 36\% 7) was determined by GC/MS with a dodecane internal standard.

Table 2, entry 5, 6 and 7: This reaction was run for $72 \mathrm{~h}$ with catalyst 5 to avoid contamination by aniline derived side products. ${ }^{5}$ Flash chromatography (5\% ethylacetate/hexane) separated 6 and 7 from the excess $N$-methylaniline in the reaction mixture. However, 6 and 7 co-eluted and chromatography conditions were not determined for their separation. A preparative HPLC separation will probably be necessary. The product was characterized as a mixture of 6 and $7 .{ }^{1} \mathrm{H}$ NMR of $6\left(\mathrm{C}_{6} \mathrm{D}_{6}, 400 \mathrm{MHz}\right) \delta 7.26$ $7.12(\mathrm{~m}, 2 \mathrm{H}, \operatorname{Ar} H), 6.85(\mathrm{~m} .1 \mathrm{H}, \operatorname{Ar} H), 6.61(\mathrm{~d}, J=7.2 \mathrm{~Hz}, 2 \mathrm{H}, \operatorname{Ar} H), 3.17\left(\mathrm{~m}, 1 \mathrm{H}, H_{a}\right), 2.62(\mathrm{~s}, 3 \mathrm{H}, N-$ Me), $2.18\left(\mathrm{bs}, 1 \mathrm{H}, H_{f}\right), 2.04\left(\mathrm{bs}, 1 \mathrm{H}, H_{c}\right), 1.53-1.33\left(\mathrm{~m}, 5 \mathrm{H}\right.$, exo- $H_{b}$, endo- $H_{b}$, exo- $H_{d}$, exo- $\left.H_{e}, s y n-H_{g}\right)$, 1.14-0.89 (m, 3H, endo- $H_{d}$, endo- $H_{e}$, anti- $\left.H_{g}\right) .{ }^{1} \mathrm{H}$ NMR of $7\left(\mathrm{C}_{6} \mathrm{D}_{6}, 400 \mathrm{MHz}\right) \delta 7.26-7.12(\mathrm{~m}, 2 \mathrm{H}, \mathrm{Ar} H)$, 6.85 (m. 2H, ArH), 3.26 (bs, 1H, NH), $2.56(\mathrm{~s}, 3 \mathrm{H}, \mathrm{N}-\mathrm{Me}) 2.37\left(\mathrm{bs}, 1 \mathrm{H}, H_{f}\right), 2.27\left(\mathrm{~m}, 1 \mathrm{H}, H_{a}\right), 2.15(\mathrm{bs}$, $\left.1 \mathrm{H}, H_{c}\right), 1.53-1.33\left(\mathrm{~m}, 5 \mathrm{H}\right.$, exo- $H_{b}$, endo- $H_{b}$, exo- $H_{d}$, exo- $H_{e}$, syn- $\left.H_{g}\right), 1.14-0.89\left(\mathrm{~m}, 3 \mathrm{H}\right.$, endo- $H_{d}$, endo$H_{e}$, anti- $\left.H_{g}\right) .{ }^{13} \mathrm{C}\left\{{ }^{1} \mathrm{H}\right\} \mathrm{NMR}$ of $6\left(\mathrm{C}_{6} \mathrm{D}_{6}, 100 \mathrm{MHz}\right) \delta 147.5,129.2,118.8,117.4,63.4,40.2,39.2,36.4$, 36.2, 30.6, 28.9. ${ }^{13} \mathrm{C}\left\{{ }^{1} \mathrm{H}\right\}$ NMR of $7\left(\mathrm{C}_{6} \mathrm{D}_{6}, 100 \mathrm{MHz}\right) \delta 146.9,131.0,126.9,125.0,118.8,110.0,41.9$, 40.6, 38.2, 37.2, 36.5, 30.4, 29.7. Mixture of 6 and 7: Anal. Calcd. C, 83.53; H, 9.51; N, 6.96. Found C, 83.29; H, 9.78; N, 7.16. GC/MS showed that the ratio of 6:7 is 1:4. Yield: $112 \mathrm{mg}(55.1 \%)$.

Table 2, entry 6, 6: Flash chromatography conditions: 5\% ethylacetate/hexane. ${ }^{1} \mathrm{H}$ NMR $\left(\mathrm{C}_{6} \mathrm{D}_{6}, 400\right.$ MHz) $\delta 7.24(\mathrm{~s}, 1 \mathrm{H}, \mathrm{p}-\mathrm{Ar} H), 6.50(\mathrm{~s}, 2 \mathrm{H}, \mathrm{o}-\mathrm{Ar} H), 3.08(\mathrm{bs}, 1 \mathrm{H}, \mathrm{NH}), 2.61\left(\mathrm{~m}, 1 \mathrm{H}, H_{a}\right), 1.95\left(\mathrm{bs}, 1 \mathrm{H}, H_{f}\right)$, $1.78\left(\mathrm{bs}, 1 \mathrm{H}, H_{c}\right), 1.32\left(\mathrm{~m}, 1 \mathrm{H}\right.$, exo- $\left.H_{b}\right), 1.21\left(\mathrm{~m}, 2 \mathrm{H}\right.$, endo- $H_{b}$, exo- $\left.H_{e}\right), 1.06\left(\mathrm{~m}, 1 \mathrm{H}\right.$, syn- $\left.H_{g}\right), 0.81(\mathrm{~m}$, $3 \mathrm{H}$, endo- $\left.H_{e}, H_{d}\right), 0.71\left(\mathrm{~m}, 1 \mathrm{H}\right.$, anti- $\left.H_{g}\right) .{ }^{13} \mathrm{C}\left\{{ }^{1} \mathrm{H}\right\} \mathrm{NMR}\left(\mathrm{C}_{6} \mathrm{D}_{6}, 100 \mathrm{MHz}\right) \delta 148.2,132.5\left(\mathrm{q},{ }^{1} \mathrm{~J}_{\mathrm{C}-\mathrm{F}}=32\right.$ Hz), 123.1, 112.2, 109.5, 56.1, 41.3, 40.4, 35.8, 35.5, 28.4, 26.1. Anal. Calcd. C, 55.73; H, 4.68; N, 4.33. Found C, 58.06; H, 4.96; N, 4.29. HRMS(EI) calcd. for $\left[\mathrm{C}_{15} \mathrm{H}_{15} \mathrm{NF}_{6}\right]^{+}: \mathrm{m} / \mathrm{z} 323.1109$, found 323.1103. A scanned image of the ${ }^{1} \mathrm{H}$ NMR spectrum is included at the end of this section. Yield: $212 \mathrm{mg}(79.6 \%)$. 
General experimental procedure for the NMR tube experiments in Table 3. A vial was charged with an accurately weighed amount of the styrene that was $\sim 35 \mathrm{mg}(\sim 0.26 \mathrm{mmol})$ for each reaction. The styrene was dissolved in $\sim 0.5 \mathrm{~mL}$ of $\mathrm{C}_{6} \mathrm{D}_{6}$. The resulting solution was mixed with 2 equivalents of the aniline $^{4}$ and $5 \mathrm{~mol} \%$ of 4 . The reaction mixture was then transferred to an NMR tube and charged with a $\mathrm{CH}_{2} \mathrm{Cl}_{2} / \mathrm{C}_{6} \mathrm{D}_{6}$ capillary tube external standard. The tube was then flame-sealed. An initial time point was taken to determine the ratio of $\mathrm{CH}_{2} \mathrm{Cl}_{2}$ to starting material before the reaction was placed in an oil bath that was previously equilibrated to the desired temperature. Yields are reported according to the ratio of the integration of the product $\mathrm{CH}_{3}$ resonance to $\mathrm{CH}_{2} \mathrm{Cl}_{2}$.

General experimental procedure for the larger scale experiments in Table 3. A vial was charged with an accurately weighed amount of the styrene that was $\sim 80 \mathrm{mg}(\sim 0.60 \mathrm{mmol})$ for each reaction. The styrene was dissolved in 4-5 mL of benzene. The resulting solution was mixed with 2 equivalents ${ }^{4}$ of the aniline and $5 \mathrm{~mol} \%$ of 4 . The reaction mixture was then transferred to a Kontes flask and sealed with a teflon stopper before being placed in an oil bath that was previously equilibrated to the desired temperature. After heating for the appropriate amount of time, the reaction mixture was then transferred to a vial and the volatile materials were removed by rotary evaporation to give an oil. The oil was then purified by flash chromatography.

Table 3, entry 3: Flash chromatography conditions: 10\% ethylacetate/hexane. Combined yield of N, O, and P: $121 \mathrm{mg}(72.4 \%)$. Ratio of 8:9:10, 25:10:65.

Table 3, entry 3, 8: ${ }^{1} \mathrm{H}$ NMR $\left(\mathrm{C}_{6} \mathrm{D}_{6}, 400 \mathrm{MHz}\right) \delta 7.09(\mathrm{~m}, 4 \mathrm{H}, \mathrm{Ar} H), 6.75(\mathrm{~d}, J=8.8 \mathrm{~Hz}, 2 \mathrm{H}, \operatorname{Ar} H)$, $6.69(\mathrm{t}, J=7.2 \mathrm{~Hz}, 1 \mathrm{H}, \operatorname{Ar} H), 6.46(\mathrm{~d}, J=7.2 \mathrm{~Hz}, 2 \mathrm{H}, \operatorname{Ar} H), 4.22(\mathrm{q}, J=6.8 \mathrm{~Hz}, 1 \mathrm{H}, \mathrm{CH}), 3.54(\mathrm{bs}, 1 \mathrm{H}$, $\mathrm{NH}), 3.28(\mathrm{~s}, 3 \mathrm{H}, \mathrm{OMe}), 1.14(\mathrm{~d}, J=6.4 \mathrm{~Hz}, 3 \mathrm{H}, \mathrm{CH}) .{ }^{13} \mathrm{C}\left\{{ }^{1} \mathrm{H}\right\} \mathrm{NMR}\left(\mathrm{C}_{6} \mathrm{D}_{6}, 100 \mathrm{MHz}\right) \delta 159.1,147.8$, 137.5, 129.3, 127.1, 117.5, 114.3, 113.8, 54.7, 52.9, 24.9. Anal. Calcd. C, 79.26; H, 7.54; N, 6.16. Found C, 79.46; H, 7.59; N, 6.45. Yield: $28.8 \mathrm{mg}(17.2 \%)$ 
Table 3, entry 3, 9: ${ }^{1} \mathrm{H}$ NMR $\left(\mathrm{C}_{6} \mathrm{D}_{6}, 400 \mathrm{MHz}\right) \delta 7.23(\mathrm{~d}, J=7.2 \mathrm{~Hz}, 1 \mathrm{H}, \mathrm{ArH}), 7.08(\mathrm{t}, J=7.6 \mathrm{~Hz}$, $1 \mathrm{H}, \operatorname{Ar} H), 6.98(\mathrm{~d}, J=6.4 \mathrm{~Hz}, 2 \mathrm{H}, \operatorname{Ar} H), 6.87(\mathrm{t}, J=7.6 \mathrm{~Hz}, 1 \mathrm{H}, \operatorname{Ar} H), 6.69(\mathrm{~d}, J=6.4 \mathrm{~Hz}, 2 \mathrm{H}, \operatorname{Ar} H)$, $6.36(\mathrm{~d}, J=7.6 \mathrm{~Hz}, 1 \mathrm{H}, \operatorname{Ar} H), 3.90$ (q, $J=7.2 \mathrm{~Hz}, 1 \mathrm{H}, \mathrm{CH}), 3.28(\mathrm{~s}, 3 \mathrm{H}, \mathrm{OMe}), 2.91$ (bs, 2H, NH), 1.45 $\left(\mathrm{d}, J=7.2 \mathrm{~Hz}, 3 \mathrm{H}, \mathrm{CH}_{3}\right) .{ }^{13} \mathrm{C}\left\{{ }^{1} \mathrm{H}\right\} \mathrm{NMR}\left(\mathrm{C}_{6} \mathrm{D}_{6}, 100 \mathrm{MHz}\right) \delta 158.9,145.3,138.2,130.5,129.0,127.7$, 119.0, 116.6, 114.7, 55.0, 40.0, 22.1. Anal. Calcd. C, 79.26; H, 7.54; N, 6.16. Found C, 79.06; H, 7.91; N, 5.97. Yield: $14.6 \mathrm{mg}(8.7 \%)$

Table 3, entry 3, 10: ${ }^{1} \mathrm{H}$ NMR $\left(\mathrm{C}_{6} \mathrm{D}_{6}, 400 \mathrm{MHz}\right) \delta 7.08(\mathrm{~d}, J=8.4 \mathrm{~Hz}, 2 \mathrm{H}, \mathrm{Ar} H), 6.99(\mathrm{~d}, J=8.4 \mathrm{~Hz}$, 2H, $\operatorname{Ar} H), 6.77(\mathrm{~d}, J=8.4 \mathrm{~Hz}, 1 \mathrm{H}, \operatorname{Ar} H), 6.33(\mathrm{~d}, J=8.4 \mathrm{~Hz}, 2 \mathrm{H}, \operatorname{Ar} H), 3.94(\mathrm{q}, J=7.2 \mathrm{~Hz}, 1 \mathrm{H}, \mathrm{CH})$, $3.31(\mathrm{~s}, 3 \mathrm{H}, \mathrm{OMe}), 2.74(\mathrm{bs}, 1 \mathrm{H}, \mathrm{N} H), 1.52\left(\mathrm{~d}, J=7.2 \mathrm{~Hz}, 3 \mathrm{H}, \mathrm{CH}_{3}\right) .{ }^{13} \mathrm{C}\left\{{ }^{1} \mathrm{H}\right\} \mathrm{NMR}\left(\mathrm{C}_{6} \mathrm{D}_{6}, 100 \mathrm{MHz}\right) \delta$ 158.4, 145.2, 139.6, 136.7, 128.8, 128.6, 115.1, 114.0, 54.7, 43.5, 22.6. Anal. Calcd. C, 79.26; H, 7.54; N, 6.16. Found C, 79.49; H, 7.75; N, 6.10. Yield: $77.9 \mathrm{mg}(46.6 \%)$

Table 3, entry 5: Flash chromatography conditions: 10\% ethylacetate/hexane.

Table 3, entry 5, 8: The proton NMR spectrum of the product of Table 3, entry 5, 8 matches the proton NMR spectrum of the product of Table 3, entry 6, 8. Yield: $139 \mathrm{mg}(74.1 \%)$.

Table 3, entry 5, 9: The proton NMR spectrum of the product of Table 3, entry 5, 9 matches the proton NMR spectrum of the product of Table 3, entry 6, 9. Yield: $15.2 \mathrm{mg}(8.1 \%)$.

Table 3, entry 6: Flash chromatography conditions: 10\% ethylacetate/hexane. Combined yield of 8 and 9: $136.8 \mathrm{mg}(85.0 \%)$. Ratio of 8:9, 20:80.

Table 3, entry 6, 8: ${ }^{1} \mathrm{H}$ NMR $\left(\mathrm{C}_{6} \mathrm{D}_{6}, 400 \mathrm{MHz}\right) \delta 7.16(\mathrm{~d}, J=8.8 \mathrm{~Hz}, 2 \mathrm{H}, \mathrm{Ar} H), 6.77(\mathrm{~d}, J=8.8 \mathrm{~Hz}$, 2H, $\operatorname{Ar} H), 6.75(\mathrm{~d}, J=6.8 \mathrm{~Hz}, 2 \mathrm{H}, \operatorname{Ar} H), 6.45(\mathrm{~d}, J=6.8 \mathrm{~Hz}, 2 \mathrm{H}, \operatorname{Ar} H), 4.23(\mathrm{q}, J=6.4 \mathrm{~Hz}, 1 \mathrm{H}, \mathrm{CH})$, $3.37(\mathrm{~s}, 3 \mathrm{H}, \mathrm{OMe}), 3.34(\mathrm{bs}, 1 \mathrm{H}, \mathrm{N} H), 3.32(\mathrm{~s}, 3 \mathrm{H}, \mathrm{OMe}), 1.22\left(\mathrm{~d}, J=6.4 \mathrm{~Hz}, 3 \mathrm{H}, \mathrm{CH}_{3}\right) .{ }^{13} \mathrm{C}\left\{{ }^{1} \mathrm{H}\right\} \mathrm{NMR}$ $\left(\mathrm{C}_{6} \mathrm{D}_{6}, 125 \mathrm{MHz}\right) \delta 159.1,152.6,142.0,137.8,128.3,127.2,115.0,114.3,55.2,54.7,53.7,25.1$. Anal. Calcd. C, 74.68; H, 7.44; N, 5.44. Found C, 74.73; H, 7.49; N, 5.44. Yield: 58.1 mg (36.1\%). 
Table 3, entry 6, 9: ${ }^{1} \mathrm{H}$ NMR $\left(\mathrm{C}_{6} \mathrm{D}_{6}, 400 \mathrm{MHz}\right) \delta 7.05(\mathrm{~s}, 1 \mathrm{H}, \mathrm{Ar} H), 7.13(\mathrm{~d}, J=8.8 \mathrm{~Hz}, 2 \mathrm{H}, \mathrm{Ar} H)$, $6.69(\mathrm{~m}, 3 \mathrm{H}, \mathrm{Ar} H), 6.32(\mathrm{~d}, J=8.4 \mathrm{~Hz}, 1 \mathrm{H}, \mathrm{ArH}), 3.96(\mathrm{q}, J=7.2 \mathrm{~Hz}, 1 \mathrm{H}, \mathrm{CH}), 3.46$ (s, 3H, OMe), 3.28 (s, 3H, OMe), $2.81(\mathrm{bs}, 2 \mathrm{H}, \mathrm{NH}), 1.46\left(\mathrm{~d}, J=7.2 \mathrm{~Hz}, 3 \mathrm{H}, \mathrm{CH}_{3}\right) .{ }^{13} \mathrm{C}\left\{{ }^{1} \mathrm{H}\right\} \mathrm{NMR}\left(\mathrm{C}_{6} \mathrm{D}_{6}, 100 \mathrm{MHz}\right) \delta$ 158.6, 153.6, 138.7, 137.8, 132.2, 128.8, 128.6, 117.3, 114.4, 112.1, 55.3, 54.7, 39.9, 22.2, 21.8. Anal. Calcd. C, 74.68; H, 7.44; N, 5.44. Found C, 74.39; H, 7.66; N, 5.83. Yield: 78.7 mg (48.9\%).

Table 3, entries 7 and 8: The yields for entries 7 and 8, in Table 3 are given as NMR yields. The isolated yields of $\mathbf{8}$ and $\mathbf{9}$ for this reaction are low due to co-elution with excess aniline. The isolated yields and characterization data for this reaction are given below. The larger scale reaction was run for 4 h at $105^{\circ} \mathrm{C}$. Flash chromatography conditions: $5 \%$ ethylacetate/hexane.

Table 3, entries 7 and 8, 8: ${ }^{1} \mathrm{H}$ NMR $\left(\mathrm{C}_{6} \mathrm{D}_{6}, 400 \mathrm{MHz}\right) \delta 7.05(\mathrm{~d}, J=8.4 \mathrm{~Hz}, 2 \mathrm{H}, \mathrm{Ar} H), 6.99(\mathrm{~d}, J=$ $6.8 \mathrm{~Hz}, 2 \mathrm{H}, \operatorname{Ar} H), 6.72(\mathrm{~d}, J=8.4 \mathrm{~Hz}, 2 \mathrm{H}, \operatorname{Ar} H), 6.02(\mathrm{~d}, J=6.8 \mathrm{~Hz}, 2 \mathrm{H}, \operatorname{Ar} H), 3.84(\mathrm{q}, J=6.4 \mathrm{~Hz}, 1 \mathrm{H}$, $\mathrm{CH}), 3.26$ (bs, $1 \mathrm{H}, \mathrm{N} H), 0.90\left(\mathrm{~d}, J=6.4 \mathrm{~Hz}, 3 \mathrm{H}, \mathrm{CH}_{3}\right) .{ }^{13} \mathrm{C}\left\{{ }^{1} \mathrm{H}\right\} \mathrm{NMR}\left(\mathrm{C}_{6} \mathrm{D}_{6}, 100 \mathrm{MHz}\right) \delta 145.4,143.2$, 132.6, 129.0, 128.7, 127.1, 122.2, 114.4, 52.5, 24.2. Anal. Calcd. C, 63.18; H, 4.92; N, 5.26. Found C, 63.49; H, 4.95; N, 5.08. Yield: $49.2 \mathrm{mg}(27.4 \%)$.

Table 3, entries 7 and 8, 9: ${ }^{1} \mathrm{H}$ NMR $\left(\mathrm{C}_{6} \mathrm{D}_{6}, 400 \mathrm{MHz}\right) \delta 7.20(\mathrm{~s}, 1 \mathrm{H}, \mathrm{Ar} H), 6.99(\mathrm{~m}, 3 \mathrm{H}, \mathrm{ArH}), 6.57$ $(\mathrm{d}, \mathrm{J}=8.4 \mathrm{~Hz}, 2 \mathrm{H}, \operatorname{Ar} H), 5.99(\mathrm{~d}, J=8.4 \mathrm{~Hz}, 1 \mathrm{H}, \operatorname{Ar} H), 3.49$ (q, J=7.2 Hz, 1H, CH), $2.64(\mathrm{bs}, 2 \mathrm{H}, \mathrm{NH})$, $1.11(\mathrm{~d}, J=7.2 \mathrm{~Hz}, 3 \mathrm{H}, \mathrm{CH}) .{ }^{13} \mathrm{C}\left\{{ }^{1} \mathrm{H}\right\} \mathrm{NMR}\left(\mathrm{C}_{6} \mathrm{D}_{6}, 100 \mathrm{MHz}\right) \delta 143.3,132.2,130.6,128.8,128.7$, 127.3, 127.2, 123.4, 117.2, 39.4, 20.9. Anal. Calcd. C, 63.18; H, 4.92; N, 5.26. Found C, 63.36; H, 4.92; N, 4.98. Yield: $69.8 \mathrm{mg}(35.1 \%)$

Table 3, entry 10, 8: Flash chromatography conditions: 5\% ethylacetate/hexane. ${ }^{1} \mathrm{H}$ NMR $\left(\mathrm{C}_{6} \mathrm{D}_{6}\right.$, $400 \mathrm{MHz}) \delta 7.15(\mathrm{~s}, 1 \mathrm{H}, \operatorname{Ar} H), 7.02(\mathrm{~d}, J=8.4 \mathrm{~Hz}, 2 \mathrm{H}, \operatorname{ArH}), 6.60(\mathrm{~d}, J=8.4 \mathrm{~Hz}, 2 \mathrm{H}, \operatorname{Ar} H), 6.44(\mathrm{~s}, 2 \mathrm{H}$, $\operatorname{ArH}), 3.69$ (q, $J=6.8 \mathrm{~Hz}, 1 \mathrm{H}, \mathrm{CH}), 3.39$ (bs, $1 \mathrm{H}, \mathrm{N} H), 0.88\left(\mathrm{~d}, J=6.8 \mathrm{~Hz}, 3 \mathrm{H}, \mathrm{C} H_{3}\right) .{ }^{13} \mathrm{C}\left\{{ }^{1} \mathrm{H}\right\} \mathrm{NMR}$ $\left(\mathrm{C}_{6} \mathrm{D}_{6}, 100 \mathrm{MHz}\right) \delta 147.6,141.9,133.4,132.1(\mathrm{q}, J=\mathrm{X}), 129.2,127.2,112.6,110.3,52.3,23.5$. Anal. 
Page S12

Calcd. C, 52.26; H, 3.29; N, 3.81. Found C, 53.23; H, 3.55; N, 3.79. HRMS(EI) calcd. for $\left[\mathrm{C}_{16} \mathrm{H}_{12} \mathrm{NClF}_{6}\right]^{+}: \mathrm{m} / \mathrm{z}$ 367.0562, found 367.0554. A scanned image of the ${ }^{1} \mathrm{H}$ NMR spectrum is included at the end of this section. Yield: $184 \mathrm{mg}(62.8 \%)$.

Table 3, entry 11, 8 and 9: Flash chromatography conditions: 10\% ethylacetate/hexane. Combined yield of $\mathbf{8}$ and 9: $165 \mathrm{mg}(81.5 \%)$. Ratio of 8:9, 40:60.

Table 3, entry 11, 8: ${ }^{1} \mathrm{H}$ NMR $\left(\mathrm{C}_{6} \mathrm{D}_{6}, 400 \mathrm{MHz}\right) \delta 6.96(\mathrm{~d}, J=8.8 \mathrm{~Hz}, 2 \mathrm{H}, \mathrm{Ar} H), 6.74(\mathrm{~m}, 3 \mathrm{H}, \mathrm{Ar} H)$, $6.65(\mathrm{~d}, J=8.4 \mathrm{~Hz}, 2 \mathrm{H}, \operatorname{Ar} H), 6.46(\mathrm{~s}, 1 \mathrm{H}, \operatorname{Ar} H), 6.14(\mathrm{~d}, J=8.4 \mathrm{~Hz}, 1 \mathrm{H}, \operatorname{Ar} H), 4.05(\mathrm{q}, J=6.4 \mathrm{~Hz}, 1 \mathrm{H}$, $\mathrm{CH}), 3.88(\mathrm{bs}, 1 \mathrm{H}, \mathrm{N} H), 3.27(\mathrm{~s}, 3 \mathrm{H}, \mathrm{OMe}), 1.06$ (d, J = 6.4 Hz, 3H, CH$).{ }^{13} \mathrm{C}\left\{{ }^{1} \mathrm{H}\right\} \mathrm{NMR}\left(\mathrm{C}_{6} \mathrm{D}_{6}, 100\right.$ MHz) $\delta 158.9,148.5,136.4,134.9,126.7,117.0,114.1,113.4,111.3,54.4,52.3,24.3$. Anal. Calcd. C, 68.83; H, 6.16; N, 5.35. Found C, 69.11; H, 6.43; N, 5.21. Yield: 54.2 mg (32.9\%).

Table 3, entry 11, 9: ${ }^{1} \mathrm{H}$ NMR $\left(\mathrm{C}_{6} \mathrm{D}_{6}, 400 \mathrm{MHz}\right) \delta 7.11(\mathrm{~d}, J=7.6 \mathrm{~Hz}, 2 \mathrm{H}, \mathrm{ArH}), 6.89(\mathrm{~d}, J=6.4 \mathrm{~Hz}$, 1H, $\operatorname{Ar} H), 6.76(\mathrm{~d}, J=7.6 \mathrm{~Hz}, 2 \mathrm{H}, \operatorname{Ar} H), 6.39(\mathrm{~s}, 1 \mathrm{H}, \operatorname{Ar} H), 6.09(\mathrm{~d}, J=6.4 \mathrm{~Hz}, 1 \mathrm{H}, \operatorname{Ar} H), 4.62(\mathrm{q}, J=$ $5.6 \mathrm{~Hz}, 1 \mathrm{H}, \mathrm{CH}), 3.31(\mathrm{~s}, 3 \mathrm{H}, \mathrm{OMe}), 2.64(\mathrm{bs}, 2 \mathrm{H}, \mathrm{NH}), 1.48(\mathrm{~d}, J=5.6 \mathrm{~Hz}, 3 \mathrm{H}, \mathrm{CH}) .{ }^{13} \mathrm{C}\left\{{ }^{1} \mathrm{H}\right\} \mathrm{NMR}$ $\left(\mathrm{C}_{6} \mathrm{D}_{6}, 100 \mathrm{MHz}\right) \delta 158.23,145.9,137.9,134.6,133.4,129.0,128.7,115.5,113.9,113.6,54.5,39.6,21.5$. Anal. Calcd. C, 68.83; H, 6.16; N, 5.35. Found C, 69.18; H, 6.47; N, 4.98. Yield: 80.2 mg (48.6\%).

Table 3, entry 12, 9: The proton NMR spectrum of this compound matches the proton NMR spectrum of the compound associated with Table 3, entry 11, 9. Yield: $127 \mathrm{mg}(70.6 \%)$.

General experimental procedure for the reactions in entries 1 and 2 in Table 4. Reactions were done on an NMR scale for Table 4, entries 1 and 2. A vial was charged with an accurately weighed amount of alkene that was $\sim 30 \mathrm{mg}(\sim 0.261 \mathrm{mmol})$ for each reaction. The alkene was then dissolved in $\sim 0.5 \mathrm{~mL}$ of $\mathrm{C}_{6} \mathrm{D}_{5} \mathrm{Cl}$. The resulting solution was mixed with 1 equivalent of aniline ${ }^{4}$ and 5 mol\% of 4 . The reaction mixture was then transferred to an NMR tube and charged with a $\mathrm{CH}_{2} \mathrm{Cl}_{2} / \mathrm{C}_{6} \mathrm{D}_{6}$ capillary tube as an external standard. The tube was flame-sealed. An initial time point was taken to determine the 
Page S13

ratio of $\mathrm{CH}_{2} \mathrm{Cl}_{2}$ to starting material before the reaction mixture was placed in an oil bath that was previously calibrated to $135^{\circ} \mathrm{C}$. Yields are reported according to the ratio of the integration of the product $\mathrm{CH}$ to the external standard, $\mathrm{CH}_{2} \mathrm{Cl}_{2}$.

In order to characterize the compounds in entries 1 and 2 of Table 4, these reactions were done on a larger scale. Unfortunately, low isolated yields resulted from co-elution with remaining aniline and contamination from dialkylation products. ${ }^{4}$ A vial was charged with an accurately weighed amount of alkene that was $\sim 80 \mathrm{mg}(\sim 1 \mathrm{mmol})$ for each reaction. The alkene was dissolved in $\sim 3 \mathrm{~mL}$ of chlorobenzene. The resulting solution was mixed with 1 equivalent of aniline ${ }^{4}$ and 5 mol\% of 4 . The reaction mixture was transferred to a reaction vessel and sealed with a Kontes stopper. The reaction mixture was then placed in an oil bath set at $135^{\circ} \mathrm{C}$ for $7 \mathrm{~d}$. The reaction mixture was then transferred to a vial and volatile materials were removed by rotary evaporation to give an oil. The oil was purified by flash chromatography ( $5 \%$ ethylacetate/hexane).

Table 4, entry 1, 14: ${ }^{1} \mathrm{H}$ NMR $\left(\mathrm{C}_{6} \mathrm{D}_{6}, 400 \mathrm{MHz}\right) \delta 7.10(\mathrm{~d}, J=7.6 \mathrm{~Hz}, 1 \mathrm{H}, \mathrm{Ar} H), 7.03(\mathrm{t}, J=7.6 \mathrm{~Hz}$, $1 \mathrm{H}, \operatorname{Ar} H), 6.81(\mathrm{t}, J=7.6 \mathrm{~Hz}, 1 \mathrm{H}, \operatorname{Ar} H), 6.43(\mathrm{~d}, J=7.6 \mathrm{~Hz}, 1 \mathrm{H}, \operatorname{Ar} H), 2.7(\mathrm{bs}, 2 \mathrm{H}, \mathrm{NH}), 2.68(\mathrm{t}, J=7.6$ $\mathrm{Hz}, 1 \mathrm{H}, \mathrm{CH}), 1.85-1.79\left(\mathrm{~m}, 2 \mathrm{H}, \mathrm{CH}_{2}\right), 1.54-1.62\left(\mathrm{~m}, 2 \mathrm{H}, \mathrm{CH}_{2}\right), 1.51-1.38\left(\mathrm{~m}, 2 \mathrm{H}, \mathrm{CH}_{2}\right) .{ }^{13} \mathrm{C}\left\{{ }^{1} \mathrm{H}\right\} \mathrm{NMR}$ $\left(\mathrm{C}_{6} \mathrm{D}_{6}, 100 \mathrm{MHz}\right) \delta 144.8,130.0,126.8,126.2,118.8,115.8,40.2,32.3,25.4$. Anal. Calcd. C, 81.94; H, 9.38; N, 8.69. Found C, 79.58; H, 9.24; N, 8.11. Yield: 9.7 mg (9.3\%).

Table 4, entry 2, 13: ${ }^{1} \mathrm{H}$ NMR $\left(\mathrm{C}_{6} \mathrm{D}_{6}, 400 \mathrm{MHz}\right) \delta 7.20(\mathrm{t}, J=7.2 \mathrm{~Hz}, 2 \mathrm{H}, \mathrm{ArH}), 6.75(\mathrm{t}, J=7.2 \mathrm{~Hz}$, $1 \mathrm{H}, \mathrm{Ar} H), 6.47(\mathrm{~d}, J=7.6 \mathrm{~Hz}, 1 \mathrm{H}, \mathrm{Ar} H), 3.29(\mathrm{bs}, 1 \mathrm{H}, \mathrm{NH}), 2.71(\mathrm{~m}, 1 \mathrm{H}, \mathrm{CH}), 1.86-0.88\left(\mathrm{~m}, 14 \mathrm{H}, \mathrm{CH}_{2}\right)$. ${ }^{13} \mathrm{C}\left\{{ }^{1} \mathrm{H}\right\}$ NMR $\left(\mathrm{C}_{6} \mathrm{D}_{6}, 100 \mathrm{MHz}\right) \delta 147.8,129.6,117.1,113.6,52.4,33.2,27.3,26.6,24.2$. Yield $3.3 \mathrm{mg}$.

Table 4, entry 2, 14: ${ }^{1} \mathrm{H}$ NMR $\left(\mathrm{C}_{6} \mathrm{D}_{6}, 400 \mathrm{MHz}\right) \delta 7.09(\mathrm{~d}, J=7.6 \mathrm{~Hz}, 1 \mathrm{H}, \mathrm{ArH}), 7.03(\mathrm{t}, J=7.6 \mathrm{~Hz}$, $1 \mathrm{H}, \operatorname{Ar} H), 6.83(\mathrm{t}, J=7.2 \mathrm{~Hz}, 1 \mathrm{H}, \mathrm{Ar} H), 6.44(\mathrm{~d}, J=7.6 \mathrm{~Hz}, 1 \mathrm{H}, \mathrm{Ar} H), 2.94(\mathrm{bs}, 2 \mathrm{H}, \mathrm{NH}), 2.58(\mathrm{~m}, 1 \mathrm{H}$, $\mathrm{CH}), 1.78-1.42(\mathrm{~m}, 14 \mathrm{H}, \mathrm{CH}) .{ }^{13} \mathrm{C}\left\{{ }^{1} \mathrm{H}\right\} \operatorname{NMR}\left(\mathrm{C}_{6} \mathrm{D}_{6}, 100 \mathrm{MHz}\right) \delta 143.5,134.0,127.0,126.6,119.0$, 
116.1, 38.4, 32.8, 27.3, 26.6, 26.4. Anal. Calcd. C, 82.70; H, 10.41; N, 6.89. Found C, 80.70; H, 10.56; N, 6.89. Yield $23.1 \mathrm{mg}(18.4 \%)$.

General experimental procedure for entries 3 and 4 in Table 4. A vial was charged with an accurately weighed amount of cyclohexadiene that was $\sim 80 \mathrm{mg}(\sim 1 \mathrm{mmol})$ for each reaction. The diene was dissolved in 3-4 $\mathrm{mL}$ of benzene. The resulting solution was mixed with 2 equivalents of the aniline ${ }^{4}$ and $5 \mathrm{~mol} \%$ of 4 . The reaction mixture was transferred to a reaction vessel and sealed with a Kontes stopper. The reaction mixture was then placed in an oil bath set at the appropriate temperature for the appropriate amount of time. The reaction mixture was then transferred to a vial and volatile materials were removed by rotary evaporation to give an oil. The oil was purified by flash chromatography (5\% ethylacetate/hexane).

Table 4, entry 3, 14: ${ }^{1} \mathrm{H}$ NMR $\left(\mathrm{C}_{6} \mathrm{D}_{6}, 500 \mathrm{MHz}\right) \delta 7.14(\mathrm{~d}, J=7.5 \mathrm{~Hz}, 1 \mathrm{H}, \operatorname{Ar} H), 7.03(\mathrm{t}, J=7.5 \mathrm{~Hz}$, 1H, $\operatorname{Ar} H), 6.79(\mathrm{t}, J=7.5 \mathrm{~Hz}, 1 \mathrm{H}, \operatorname{Ar} H), 6.40(\mathrm{~d}, J=7.5 \mathrm{~Hz}, 1 \mathrm{H}, \operatorname{Ar} H), 5.79(\mathrm{~m}, 1 \mathrm{H}, \mathrm{CH}), 5.69(\mathrm{dd}, J=$ $10 \mathrm{~Hz}, J=2.5 \mathrm{~Hz}, 1 \mathrm{H}, \mathrm{CH}), 3.21(\mathrm{~m}, 1 \mathrm{H}, \mathrm{CH}), 3.09$ (bs, 2H, NH), 1.89 (m, 2H, $\left.\mathrm{CH}_{2}\right), 1.77\left(\mathrm{~m}, 1 \mathrm{H}, \mathrm{CH}_{2}\right)$, $1.68(\mathrm{~m}, 1 \mathrm{H}, \mathrm{CH}), 1.57\left(\mathrm{~m}, 1 \mathrm{H}, \mathrm{CH}_{2}\right), 1.41\left(\mathrm{~m}, 1 \mathrm{H}, \mathrm{CH} \mathrm{H}_{2}\right) .(\mathrm{m}, 1 \mathrm{H}, \mathrm{CH}) .{ }^{13} \mathrm{C}\left\{{ }^{1} \mathrm{H}\right\} \operatorname{NMR}\left(\mathrm{C}_{6} \mathrm{D}_{6}, 125\right.$ MHz) $\delta 144.6,130.4,129.6,129.3,128.9,127.4,118.7,116.2$. Anal. Calcd. C, 83.19; H, 8.73; N, 8.08. Found C, 83.16; H, 8.68; N, 8.21. Yield 86.2 mg (52.2\%).

Table 4, entry 4, 13: ${ }^{1} \mathrm{H} N M R\left(\mathrm{C}_{6} \mathrm{D}_{6}, 500 \mathrm{MHz}\right) \delta 7.20(\mathrm{~s}, 1 \mathrm{H}, \operatorname{Ar} H), 6.49(\mathrm{~s}, 2 \mathrm{H}, \operatorname{Ar} H), 5.59(\mathrm{dd}, J=$ $10 \mathrm{~Hz}, J=3.2 \mathrm{~Hz}, 1 \mathrm{H}, \mathrm{C} H), 5.33(\mathrm{dd}, J=10 \mathrm{~Hz}, J=3.2 \mathrm{~Hz}, 1 \mathrm{H}, \mathrm{CH}), 3.38(\mathrm{bs}, 1 \mathrm{H}, \mathrm{NH}), 3.23(\mathrm{~m}, 1 \mathrm{H}$, $\mathrm{CH}), 1.69\left(\mathrm{~m}, 2 \mathrm{H}, \mathrm{CH}_{2}\right), 1.38-1.12(\mathrm{~m}, 4 \mathrm{H}, \mathrm{CH}) .{ }^{13} \mathrm{C}\left\{{ }^{1} \mathrm{H}\right\} \mathrm{NMR}\left(\mathrm{C}_{6} \mathrm{D}_{6}, 125 \mathrm{MHz}\right) \delta 148.0,132.5(\mathrm{q}, J=$ $41 \mathrm{~Hz}$ ). HRMS(EI) calcd. for $\left[\mathrm{C}_{14} \mathrm{H}_{12} \mathrm{NF}_{6}\right]^{+}: \mathrm{m} / \mathrm{z}$ 309.0948, found 309.0952. Yield: $47.8 \mathrm{mg}$ (19.7\%). The yield of this reaction is low due to contamination of the product mixture by double alkylation products. A scanned ${ }^{1} \mathrm{H}$ NMR spectrum is given at the end of this section. 


\section{Supporting Mechanistic Discussion}

\section{A. Use of 2,6-Di-t-butyl-4-methylpyridine as a Proton-Trapping Reagent:}

As described in the manuscript, a mixture of norbornene ( 1 equiv), aniline (5 equiv), and 4 (5 mol\%) provides a $60 \%$ yield of $\mathbf{2}$ and $\mathbf{3}$ in a 1:1 ratio. If $5 \mathrm{~mol}^{2} \mathrm{NEt}_{3}$ is added to this mixture no reaction is observed. This observation is consistent with the difference in $\mathrm{pK}_{\mathrm{a}}$ values between anilinium (4.6) ${ }^{6}$ and $\mathrm{HNEt}_{3}{ }^{+}(10.6)^{6}$ and suggests that the reaction is a proton-catalyzed process.

Scheme S1

A)

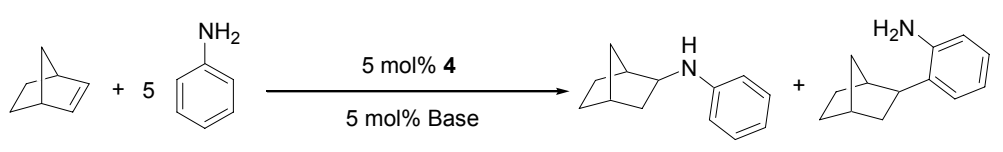

B)
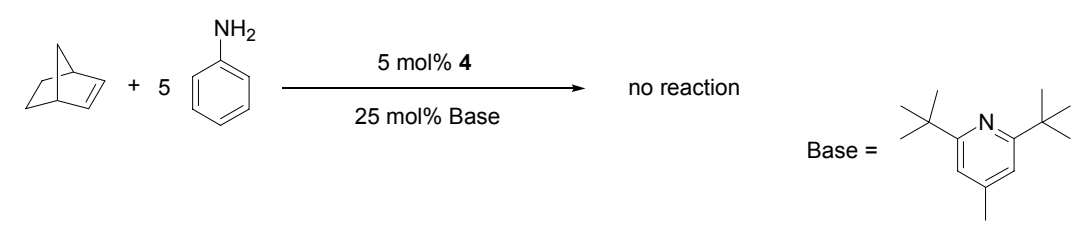

The Lewis base, 2,6-di-t-butyl-4-methylpyridine (2,6-di-t-butyl-pyridinium $\left.\mathrm{pK}_{\mathrm{a}}=4.95\right)$, is also commonly used as a proton-trapping reagent. The bulky ortho-substitution of 2,6-di-t-butyl-4methylpyridine makes this compound highly unlikely to coordinate to a metal center and thus it should be selective for proton association. ${ }^{7}$ The addition $5 \mathrm{~mol} \%$ 2,6-di-t-butyl-4-methylpyridine did not effect the yield or product ratio of the reaction shown in Scheme S1A; however, when 25 mol\% 2,6-di-t-butyl-4methylpyridine were added to the reaction mixture no reaction was observed after $24 \mathrm{~h}$ at $135{ }^{\circ} \mathrm{C}$ (Scheme S1B).

The difference in effectiveness of $\mathrm{NEt}_{3}$ and 2,6-di-t-butyl-4-methylpyridine as proton-trapping reagents agrees with the difference between the respective $\mathrm{pK}_{\mathrm{a}}$ values of these compounds. The significantly higher basicity of $\mathrm{NEt}_{3}$ results in preferential formation of $\mathrm{HNEt}_{3}{ }^{+}$over $\mathrm{PhNH}_{3}{ }^{+}$and prevents the desired hydroamination/hydroarylation reaction from proceeding. The similar acidities of 2,6-di-tbutylpyridinium and $\mathrm{PhNH}_{3}{ }^{+}$suggest that the equilibrium constant between protonated aniline and protonated di-t-butyl-4-methylpyridine is close to 1 and that there is a significant amount of competition 
between these two reagents for the reactive proton. When additional equivalents of pyridine are added to the reaction mixture, the equilibrium is shifted towards pyridine protonation, shutting down the hydroamination/hydroarylation reaction.

The observation that five equivalents of 2,6-di-t-butyl-4-methylpyridine inhibits the hydroamination/hydroarylation reaction of aniline and norbornene catalyzed by $\mathbf{4}$, provides evidence that this reaction is proton-catalyzed and does not involve trace amounts of metal reagents.

\section{B. Clarification of Yields Given in Tables 1, 2, and 3.}

The yields given in Tables 1, 2, and 3 are combined yields of the isomeric products. These yields are based on the mass of the norbornene used for the reaction and the amount of product observed or isolated.

\section{Proposed mechanism for proton-catalyzed hydroamination and hydroarylation of styrenes.}

The substituent trends and exclusively Markovnikov regioselectivity that are observed for the styrene hydroamination/hydroarylation reactions illustrated in Table 3 suggest that these transformations are proceeding through cationic intermediates and reaction mechanisms analogous to acid-catalyzed hydration. A proposed mechanism for the proton-catalyzed hydroamination and hydroarylation of styrene substrates is shown in Scheme S2. In this proposed mechanism, protonation of the styrene by an anilinium salt produces a carbocationic intermediate that can then be attacked by either the nitrogen or the aryl nucleophilic sites of aniline. The nucleophilic attack produces anilinium or arenium intermediates that can then be deprotonated by aniline to reform the anilinium catalyst and give the observed hydroamination and hydroarylation products. 


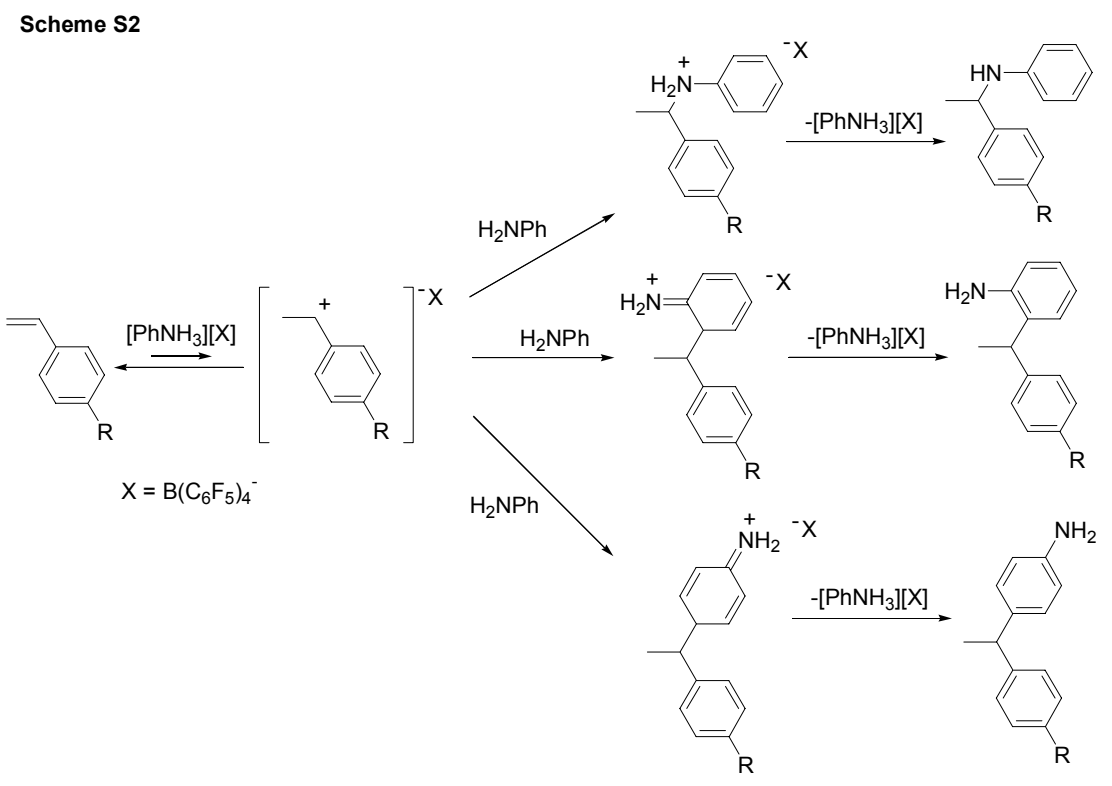

\section{Proposed mechanism for proton-catalyzed hydroamination and hydroarylation of norbornene.}

The hydroamination and hydroarylation reactions of norbornene and the other cyclic alkenes shown in Table 4 may also be proceeding through a carbocationic mechanism similar to that proposed for the styrene substrates (Scheme S3). The positively charged intermediates of these reactions, however, are higher in energy than their styrene analogues. This coincides with the higher temperatures required to perform these reactions.

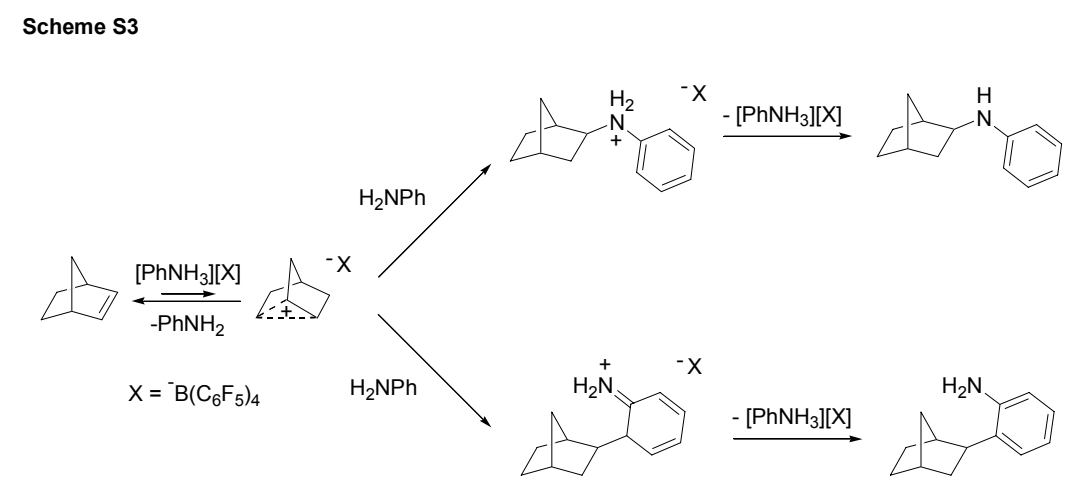

The presence of charged intermediates in a nonpolar solvent such as benzene is likely to cause aggregation. Aggregation may be particularly important for the reactions of norbornene and other cyclic alkenes in order to stabilize the higher energy carbocationic intermediates associated with these 
transformations. Additionally, aggregation may explain the ortho-selectivity of these reactions. ${ }^{8}$ Further details of these mechanisms will need to be determined by careful mechanistic studies.

\section{E. The Role of the $B\left(\mathrm{C}_{6} \mathrm{~F}_{5}\right)_{4}{ }^{-}$Anion in Promoting Alkene Protonation:}

As discussed above, proton-catalyzed hydroamination and hydroarylation requires protonation of the alkene with an anilinium salt. The equilibrium constant for this process is small and protonation of aniline will occur preferentially under the reaction conditions. The dramatic anilinium counterion effect observed for the hydroamination/hydroarylation reaction discussed in this communication suggests that the weakly-coordinating $\mathrm{B}\left(\mathrm{C}_{6} \mathrm{~F}_{5}\right)_{4}{ }^{-}$anion increases the effective acidity of the anilinium salt to allow for sufficient alkene protonation to lead to further reactivity.

\section{F. Prevention of Alkene Polymerization:}

The protonated alkene intermediate proposed for the hydroamination/hydroarylation reactions can potentially react with another molecule of alkene in order to form oligomers or polymers. This type of reactivity is observed if the aniline to alkene ratio is $1: 1$. The $2: 1$ or $5: 1$ ratio of aniline:alkene prevents this type of undesirable side reaction and provides the observed hydroamination and hydroarylation products.

\section{References:}

(1) Alaimo, P. J.; Peters, D. W.; Arnold, J.; Bergman, R. G. J. Chem. Ed. 2001, 78, 64.

(2) Lambert, J. B.; Zhang, S. Z.; Ciro, S. M. Organometallics 1994, 13, 2430.

(3) Seligson, A. L.; Trogler, W. C. Organometallics 1993, 12, 744.

(4) Five equivalents of the aniline were used in the catalytic hydroamination/hydroarylation reactions with norbornene. This prevented contamination of the desired products with products resulting from the addition of two equivalents of styrene to a single aniline. In the reactions of styrene and other cyclic alkenes, however, excess aniline confounded product isolation due to co-elution during chromatography. In order to facilitate isolation and characterization of these products only two equivalents of the aniline were used for the styrene and cyclohexadiene reactions and only a stoichiometric amount of an aniline was 
used for the reactions of cyclopentene and cyclooctene. This decrease in the aniline: styrene ratio led to increased amounts of double alkylation products in some cases.

(5) The use of $\mathrm{PhNH}_{3} \mathrm{~B}\left(\mathrm{C}_{6} \mathrm{~F}_{5}\right)_{4}$ as a catalyst introduces 5 mol\% aniline into a reaction mixture which can independently react with the substrate. In the reaction of $N$-methylaniline with norbornene the side products derived from aniline were inseparable from the desired product. Therefore, $\mathbf{5}$ was used as a catalyst to prevent this contamination. Because $\mathbf{5}$ is a less efficient catalyst than $\mathbf{4}$, the reaction was run for $72 \mathrm{~h}$. As a control experiment, the reaction of $p$-chloroaniline with norbornene was also catalyzed by 5 giving an almost identical isolated yield to when $\mathbf{4}$ was used as a catalyst for this reaction.

(6) The $\mathrm{pK}_{\mathrm{a}}$ values that are given are aqueous values. These values will change in nonpolar solvents but we are assuming that the trends will be the same.

(7) Kanner, B. Heterocycles 1982, 18, 411.

(8) For other examples of ortho- and para-selective Friedel-Crafts reactions see: Pearson, D. E.; Buehler, C. A. Synthesis 1971, 455. 


\section{Scanned Spectra:}

Table 2, entry $6, \mathrm{~N}$ :

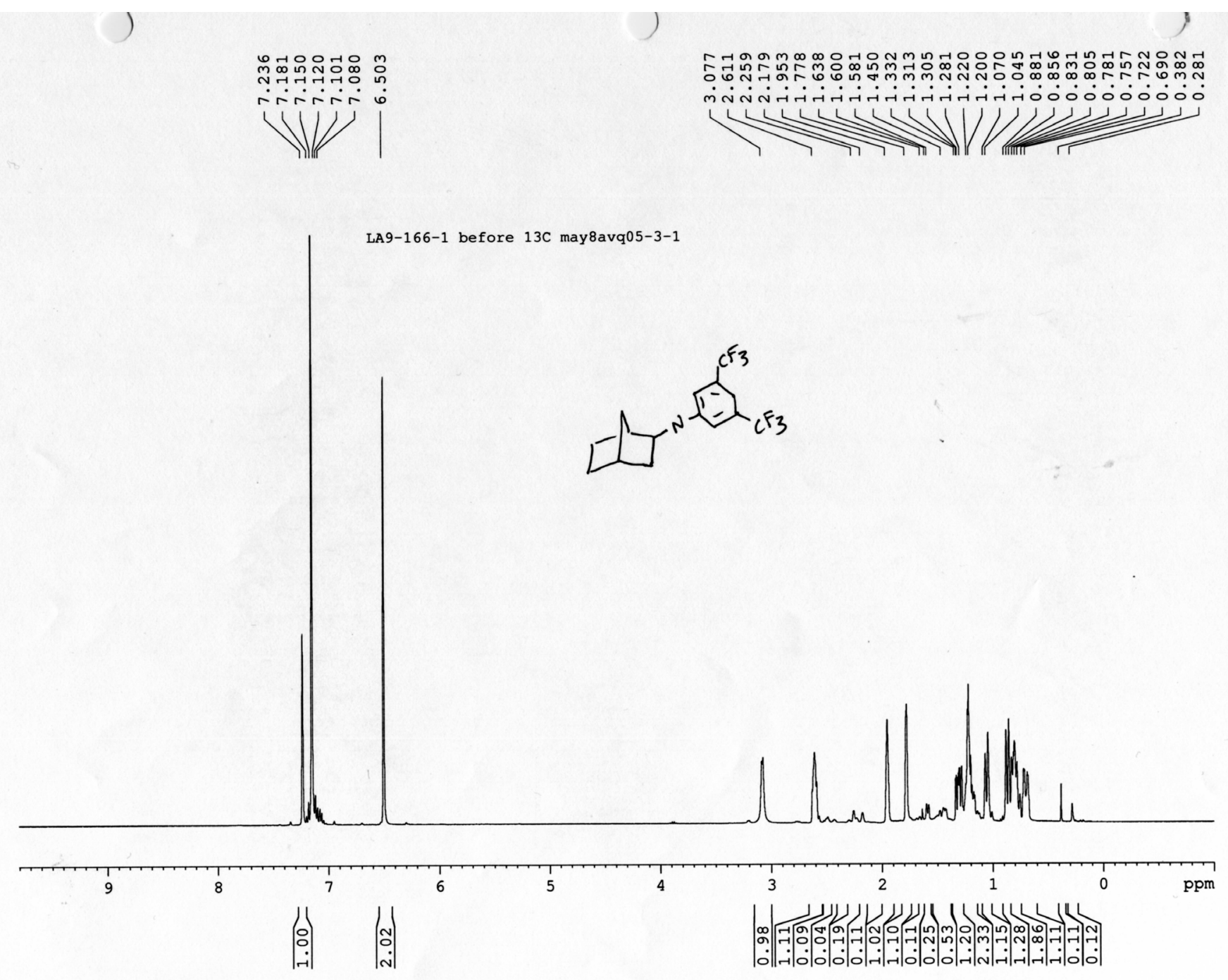


Table 3, entry 10, N:

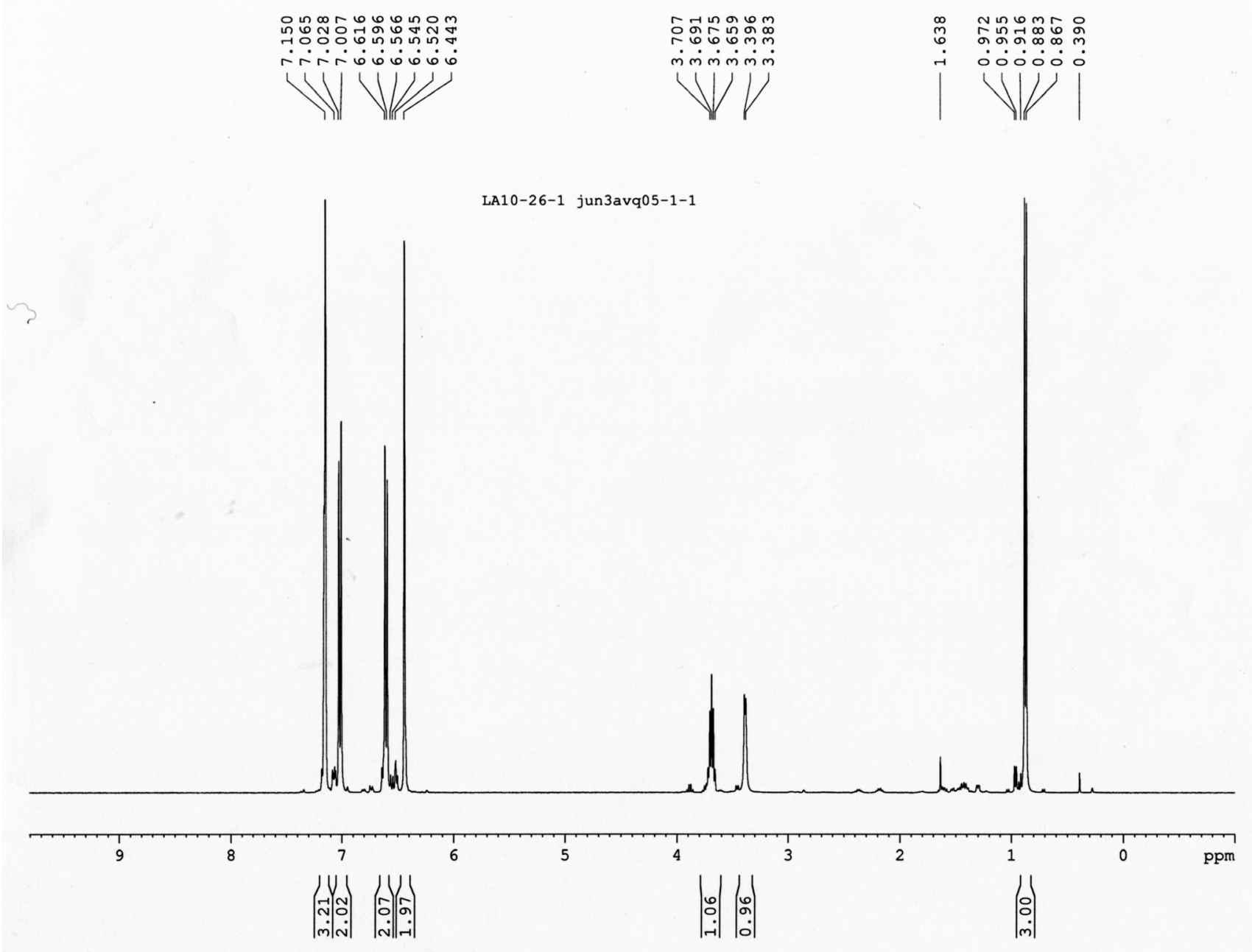


Table 4, entry 4, N:

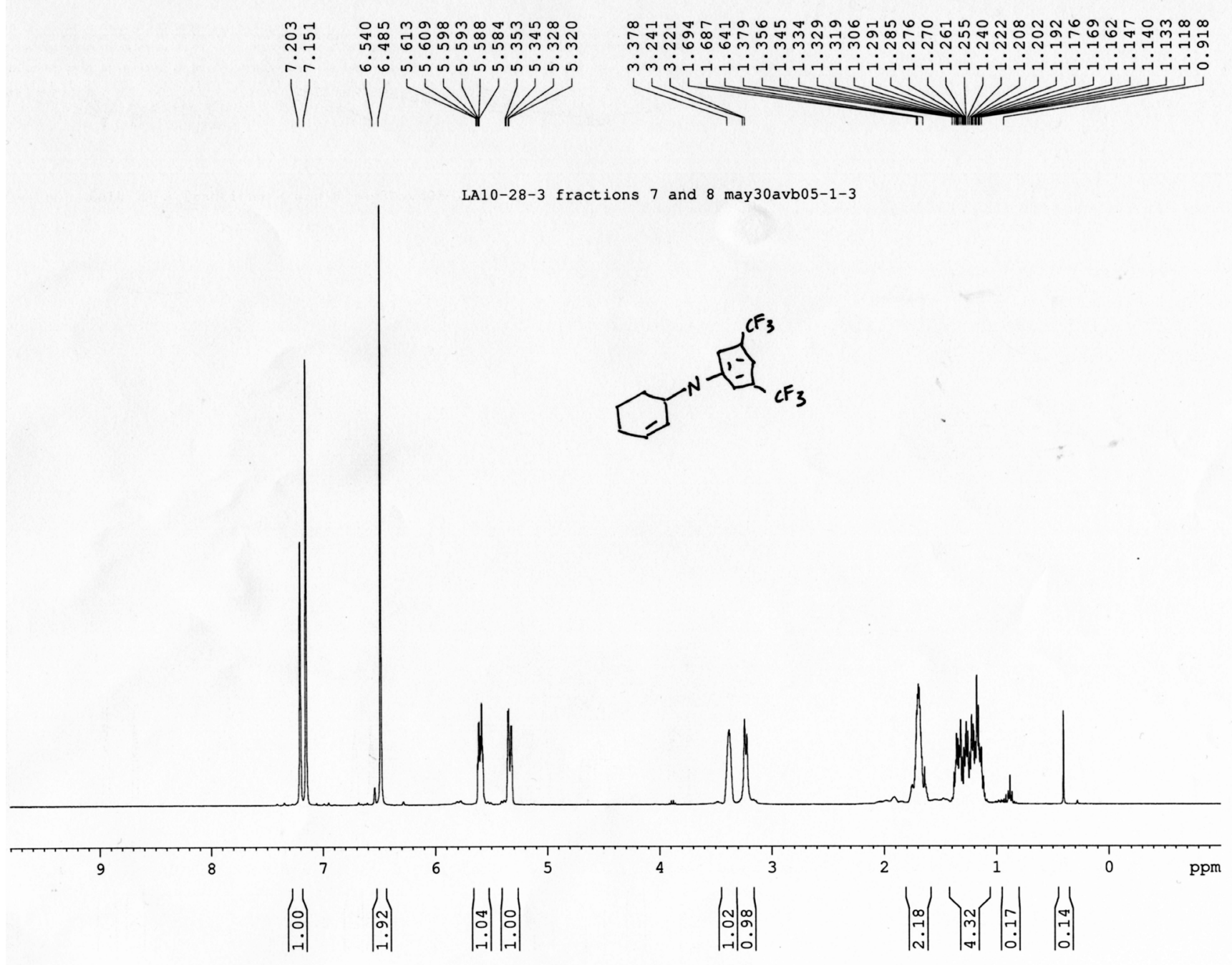

